\title{
Reanatomização do sorriso com uso de resina composta: relato de caso
}

Smile reanatomization using composite resin: case report

Reanomatización de la sonrisa con uso de resina compuesta: relato de caso Martha Gerusa da Silva LIMA ${ }^{1}$

Fabíola Gabriellen de Barros BRITO ${ }^{1}$ José Henrique de Araújo $\mathbf{C R U Z}^{1}$

Luanna Abílio Diniz Melquíades de MEDEIROS ${ }^{2}$ Elizandra Silva da PENHA ${ }^{2}$

Camila Helena Machado da Costa FIGUEIREDO² Gymenna Maria Tenório GUÊNES ${ }^{2}$

${ }^{1}$ Acadêmico(a) do Curso de Graduação em Odontologia. Centro de Saúde e Tecnologia Rural, Universidade Federal de Campina Grande, UFCG 58708-110 Patos/PB, Brasil ${ }_{2}^{2}$ Professora Doutora do Curso de Graduação em Odontologia. Centro de Saúde e Tecnologia Rural, Universidade Federal de Campina Grande, UFCG $58708-110$ Patos/PB, Brasil

\section{Resumo}

Introdução: Os pacientes têm exigido cada vez mais da odontologia estética para solucionar casos de desarmonia do sorriso, como alterações de cor, forma e tamanho. Com o avanço dos materiais dentários e aperfeiçoamento das técnicas é possível tratar casos estéticos com mínimos desgastes dentários e ter resultados excelentes. Cabe ao cirurgião-dentista optar por um tratamento que devolva saúde e função além da estética. Objetivo: Realizar reanatomização do sorriso por meio de facetas diretas em resina composta, como também mostrar que é possível alcançar sucesso clínico usando um material mais barato como a resina composta. Relato do caso: Paciente sexo feminino, 22 anos, apresentou-se à Clínica Escola de Odontologia da UFCG buscando melhorar a estética do seu sorriso. O material de escolhido foi resina composto por ser mais viável financeiramente em relação às cerâmicas, ter execução mais simples, necessitar de pouco ou nenhum desgaste e também ser reversível. A técnica usada foi Facetas diretas em resina composta; para realiza-lo foram imprescindíveis etapas pré-operatórias de gengivectomia, clareamento dental, enceramento diagnóstico para posteriormente realizar-se os desgastes. Utilizou-se guia de silicone para nortear os aumentos incisais, condicionamento ácido com ácido fosfórico a 37\%, aplicação do sistema adesivo e realização dos incrementos de compósitos com posterior acabamento e polimento. Conclusão: A colaboração da paciente, um bom planejamento do caso, execução adequada da técnica e controle e manutenção periódica, proporcionaram uma estética dentária dentro dos padrões tão valorizados atualmente e aumento da sua autoestima.

Descritores: Estética Dentária; Facetas Dentárias; Resinas Compostas.

\section{Abstract}

Introduction: Patients have increasingly required aesthetic dentistry to solve cases of smile disharmony, such as changes in color, shape and size. With the advancement of dental materials and improved techniques it is possible to treat aesthetic cases with minimal dental wear and have excellent results. It is up to the dentist to choose a treatment that returns health and function beyond esthetics. Objective: To perform a smile reanatomization through direct facets of composite resin, as well as to show that it is possible to achieve clinical success using a cheaper material such as composite resin. Case report: A 22-year-old female patient presented to the UFCG School of Dentistry Clinic seeking to improve the aesthetics of her smile. The chosen material was resin composed of being more financially feasible in relation to ceramics, having simpler execution, requiring little or no wear and also being reversible. The technique used was Direct facets in composite resin; to perform it were essential preoperative steps of gingivectomy, dental bleaching, diagnostic waxing for later wear. It was used a guide of silicone to guide the incisal increases, acid conditioning with $37 \%$ phosphoric acid, application of the adhesive system and realization of the increments of composites with later finishing and polishing. Conclusion: Patient collaboration, good case planning, adequate technique execution, and periodic maintenance and control have provided aesthetic dentistry within today's valued standards and increased self-esteem.

Descriptors: Esthetics, Dental; Dental Veneers; Composite Resins.

\section{Resumen}

Introducción: Los pacientes han exigido cada vez más la odontología estética para solucionar casos de desarmonía de la sonrisa, como cambios de color, forma y tamaño. Con el avance de los materiales dentales y el perfeccionamiento de las técnicas es posible tratar casos estéticos con mínimos desgastes dentales y tener resultados excelentes. Es el cirujano dentista optar por un tratamiento que devuelva salud y función además de la estética. Objetivo: Realizar reanatomización de la sonrisa por medio de facetas directas en resina compuesta, como también mostrar que es posible alcanzar éxito clínico usando un material más barato como la resina compuesta. Relato del caso: Paciente sexo femenino, 22 años, se presentó a la Clínica Escuela de Odontología de la UFCG buscando mejorar la estética de su sonrisa. El material de elección fue resina compuesto por ser más viable financieramente en relación a las cerámicas, tener ejecución más simple, necesitar de poco o ningún desgaste y también ser reversible. La técnica utilizada fue Facetas directas en resina compuesta; para realizarlo fueron imprescindibles etapas preoperatorias de gingivectomía, blanqueamiento dental, encerado diagnóstico para posteriormente realizar los desgastes. Se utilizó guía de silicona para orientar los aumentos incisales, condicionamiento ácido con ácido fosfórico a 37\%, aplicación del sistema adhesivo y realización de los incrementos de composites con posterior acabado y pulido. Conclusión: La colaboración de la paciente, una buena planificación del caso, ejecución adecuada de la técnica y control y mantenimiento periódico, proporcionaron una estética dental dentro de los patrones tan valorados actualmente y aumento de su autoestima.

Descriptores: Estética Dentária; Coronas con Frente Estético; Resinas Compuestas.

\section{INTRODUÇÃO}

Os pacientes exigem cada vez mais da odontologia estética. Detalhes como cor, forma, posição e tamanho são cada vez mais observados por maior parte dos pacientes, isso influencia na autoestima e interações interpessoais. Assim o trabalho do cirurgião-dentista nos dias atuais vai além da reabilitação da função dentária ${ }^{1,2}$.

Pode-se observar que os pacientes têm buscado no consultório odontológico por procedimentos estéticos que melhorem a harmonia dentofacial exigindo principalmente que esses procedimentos mimetizem a naturalidade. Dessa forma, a odontologia estética vem se destacando e crescendo a cada dia ${ }^{3}$.

Além de ser procurado para reabilitar lesões simples, o cirurgião-dentista recebe frequentemente casos de reabilitações extensas que necessitam do uso de mais de uma especialidade sendo necessários planejamento e abordagem multidisciplinar ${ }^{4}$.

$\mathrm{O}$ avanço dos materiais dentários junto ao 
aperfeiçoamento das técnicas restauradoras minimamente invasivas com uso de resina composta tem permitido o cirurgião-dentista realizar restaurações estéticas em dentes anteriores com boa eficiência, pois permite maior mimetização da naturalidade, preservação de estrutura dentária hígida reabilitando o sorriso estética e funcionalmente ${ }^{5}$.

As facetas dentárias são alternativas para reestabelecer a função e estética em dentes anteriores e estão divididas em dois grupos: diretas e indiretas. As diretas são feitas exclusivamente pelo cirurgiãodentista, que fará a inserção dos compósitos resinosos diretamente na estrutura dentária após a mesma ter sido preparada para receber esse material. Já as indiretas precisam de um trabalho em conjunto entre o dentista e um laboratório de prótese ${ }^{6}$.

Logo, para obter previsibilidade do resultado e consequentemente sucesso clínico, é fundamental um planejamento individualizado, levando em consideração as características e anseios do paciente para escolher os materiais e técnicas que mais se adequem a cada caso. $\mathrm{O}$ uso de fotografias e modelos de enceramento diagnóstico contribui nesse processo $^{7,8}$.

Assim sendo, o intuito do presente trabalho será a apresentação de um caso clínico, no qual se propôs a reabilitação do sorriso de uma paciente por meio de reanatomização com resina composta trazendo-lhes melhorias na qualidade de vida ao reestabelecer a harmonia do sorriso.

\section{CASO CLÍNICO}

Paciente de 22 anos, feoderma, sexo feminino, procurou a clínica escola de Odontologia da Universidade Federal de Campina Grande com objetivo de melhorar a estética do seu sorriso (Figura 1). Após anamnese e exame clínico foi observado leve inflamação gengival e excesso de resina composta entre os incisivos centrais superiores. Inicialmente foi realizado uma adequação do meio bucal com desgaste do excesso de resina com pontas diamantadas associado à raspagem e alisamento corono-radicular para se obter a desinflamação gengival.

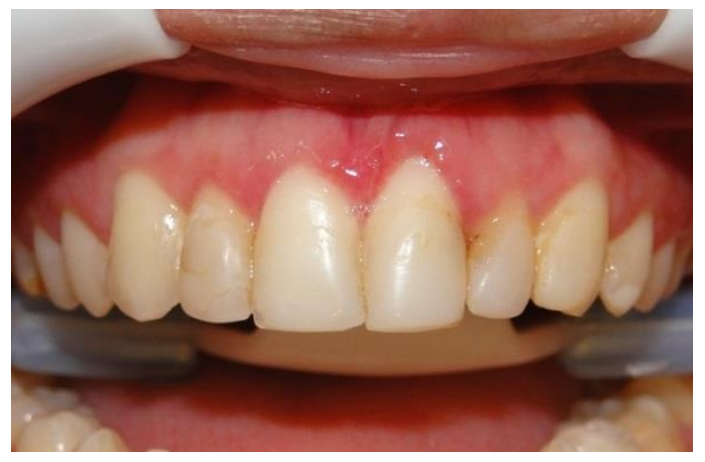

Figura 1: Aspecto inicial do sorriso.

Nesta mesma sessão foi realizada a moldagem para a posterior confecção do enceramento diagnóstico, a fim de potencializar positivamente o planejamento. Subsequentemente à adequação do meio bucal, e desinflamação da gengiva, foi realizada a cirurgia gengival para aumento de coroa clínica nos elementos 11, 12,21 e 22 para melhorar a estética dental. Após a recuperação do procedimento periodontal, optou-se por remover as resinas antigas e realizar novas restaurações com resina composta nos elementos 11 , $12,13,14,15,21,22,23,24$ e 25.

Os procedimentos clínicos e laboratoriais foram divididos em etapas. Primeiramente foram feitos registros fotográficos para ajudar a guiar o planejamento, em seguida, foi feita a moldagem e confecção de modelo de estudo, o qual foi encaminhado para um laboratório de prótese dentária para realização de enceramento diagnóstico com todos os dados relativos ao caso e com base no objetivo a ser alcançado.

Paralelamente realizou-se clareamento dental assistido em consultório com clareador à base de peróxido de hidrogênio a 35\% (Whiteness HP Blue ${ }^{\circledR}$ - FGM Produtos Odontológicos, Joinville-SC, Brasil). Apenas uma sessão foi realizada, pois a paciente apresentou muita sensibilidade devido ao seu baixo limiar de dor. Após o enceramento pronto (Figuras 2 e 3), foi feito um molde da região encerada utilizando silicona de adição (Adsil Putty Soft @ Coltene, Rio de Janeiro-RJ, Brasil) para ser usado como guia no momento das restaurações (Figura 4).

Posteriormente ao planejamento com uso de um tratamento restaurador direto com resinas compostas, iniciaram-se os desgastes da resina composta desfavorável no elemento dental necessários com ponta diamantada 2135 (KG Sorensen ${ }^{\circledR}$ Cotia, SP) em alta rotação nos elementos 11, 12, 13, 21, 22 e 23 (Figura 5). Depois de serem feitos os desgastes foi realizado profilaxia com pedra pomes e escova de Robinson (Microdont ${ }^{\circledR}$ - São Paulo, SP) em baixa rotação (Figura 6).

Em seguida à profilaxia foi selecionada a cor A1 da resina Z350® (3M ESPE - São Paulo, SP) para ser utilizada em todas as facetas diretas, depois mediante isolamento relativo com rolete de algodão foi feito condicionamento ácido total com gel de ácido fosfórico a 37\% por 15 segundos (Figura 7), o local foi abundantemente lavado com água e seco com leve jato de ar, mantendo-se a dentina com aspecto acetinado.

Foi aplicado sistema adesivo Adper Single Bond ${ }^{\circledR}$ (3M ESPE - Sumaré, SP) e fotopolimerizado por 40s. Com o dente preparado e escolhida a cor foi realizada a inserção da resina composta Filtek Z350 XT, na cor A1B (3M ESPE) com espátula de resina Almore, usando também a guia de silicone para facilitar a execução (Figura 8).

Ao fim dos incrementos de resina composta foi realizado acabamento com ponta diamantada 2135 F, 2135 FF, 3118 e tiras de lixa. Na sessão 
seguinte foram feitos os acréscimos nos dentes 14, 15, 24 e 25, seguindo o mesmo protocolo anteriormente aplicado.

Ao finalizar as restaurações foram realizados acabamento e polimento final com pontas diamantadas, tiras de lixa, discos de lixa (Figura 9), discos Sof-Lex Pop On® (3M ESPE - São Paulo, SP), borrachas siliconadas, escova de carbeto de silício Astrobrush ${ }^{\circledR}$ (Ivoclair Vivadent - Barueri, SP) e por fim feltro e pasta diamantada obtendo-se superfície lisa e brilhante como resultado do polimento (Figuras 10 e 11).

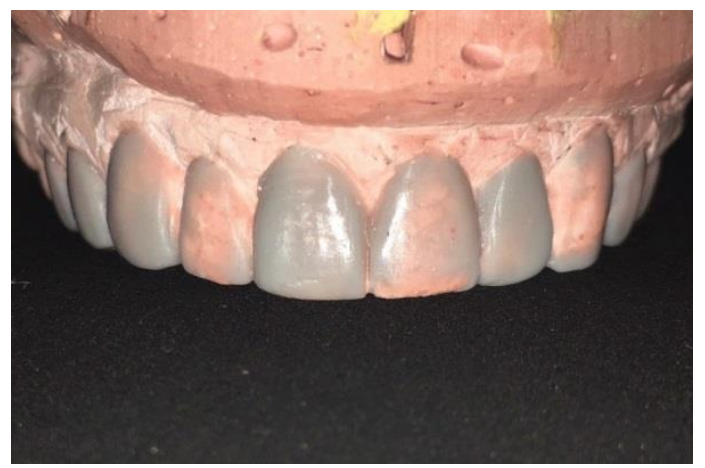

Figura 2: Enceramento diagnóstico sob vista vestibular.

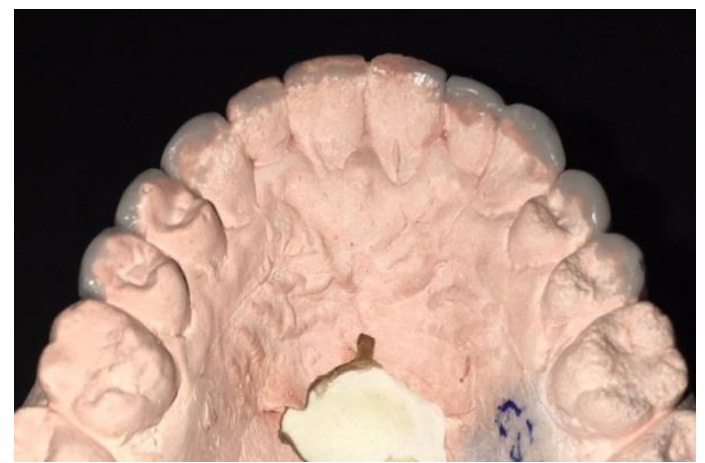

Figura 3: Enceramento diagnóstico sob vista palatina.

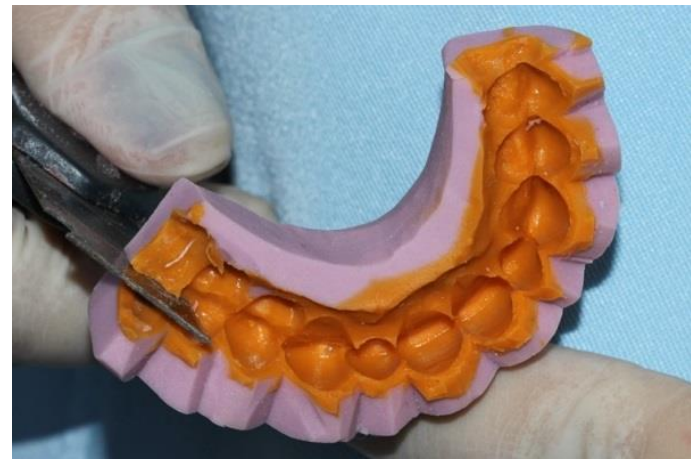

Figura 4: Guia de silicone.

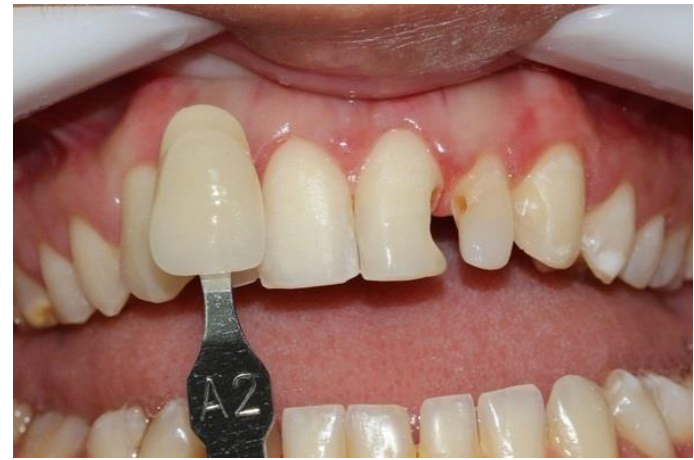

Figura 5: Seleção da cor.

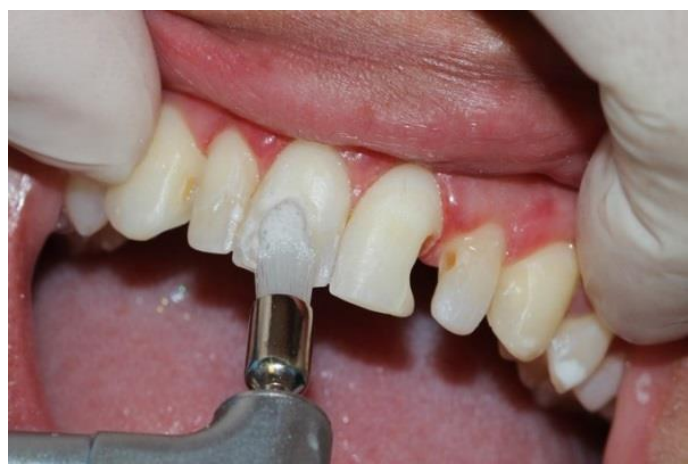

Figura 6: Profilaxia com pedra pomes.

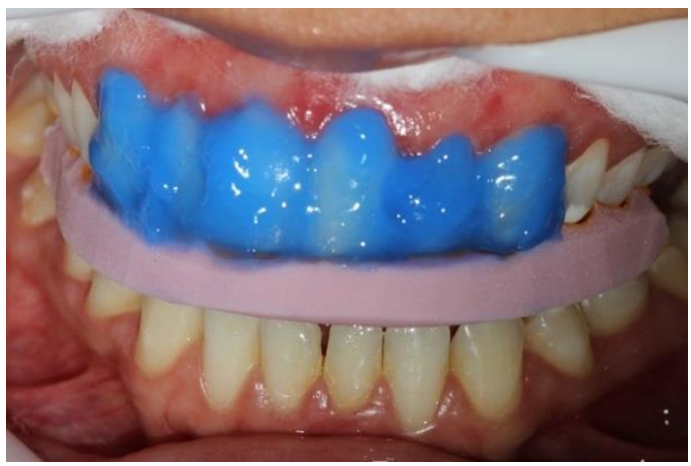

Figura 7: Aplicação do ácido fosfórico a 37\%.

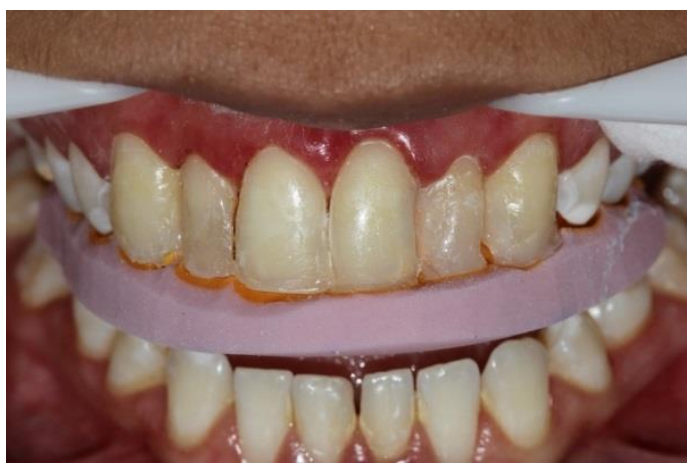

Figura 8: Incrementos de resina composta com guias de silicone.

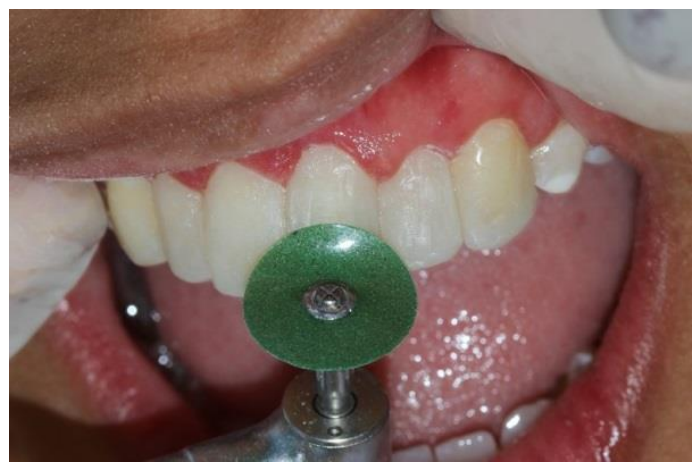

Figura 9: Acabamento com discos de lixa.

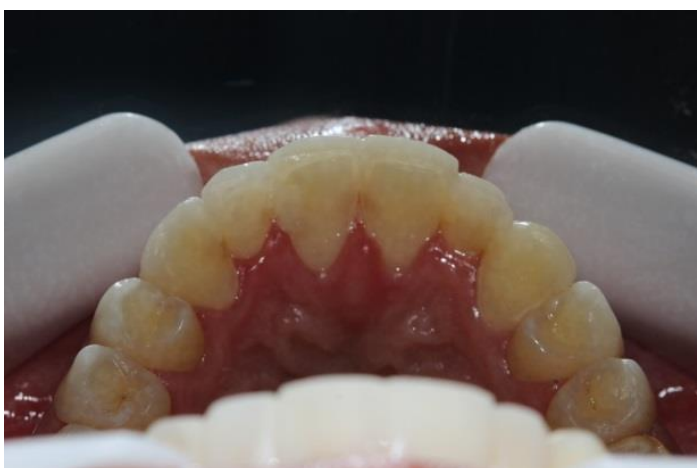

Figura 10: Vista oclusal do resultado final. 


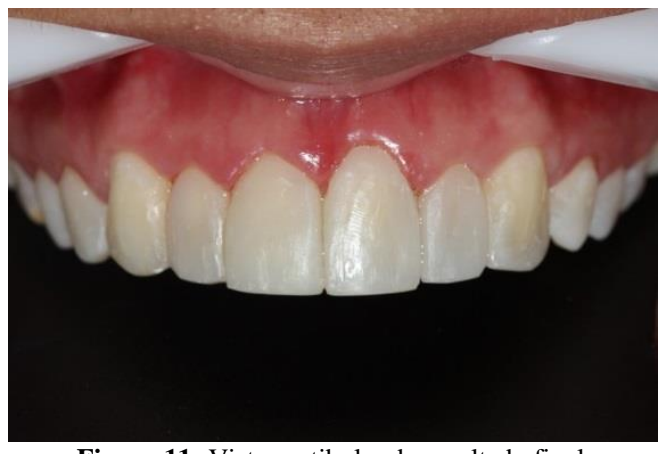

Figura 11: Vista vestibular do resultado final.

\section{DISCUSSÃO}

A busca por tratamentos que permitam um sorriso estético, saudável e harmônico tem crescido a cada dia, exigindo dos profissionais de odontologia maior busca por tratamentos que satisfaçam as expectativas dos pacientes e que sejam acessíveis de acordo com o potencial financeiro individual.

Há vários estudos que revelam insatisfação de grande parte das pessoas não apenas com a forma e alinhamento dos seus dentes, mas também com relação à cor dos mesmos, o que faz desta uma característica bastante associada à procura por tratamentos estéticos ${ }^{9}$. Esse descontentamento também foi relatado pela paciente, o que levou à realização de clareamento dental assistido em consultório como primeiro passo do tratamento a fim de promover estética branca dos dentes e aplicação dos compósitos.

Um dos fatores mais desejados pelos profissionais e pacientes é o sucesso clínico associado à longevidade. Apesar das restaurações cerâmicas apresentarem maior longevidade, as restaurações diretas em resina composta podem durar até 10 anos quando bem executadas ${ }^{10}$.

Associado a essa longevidade, que junto à vontade do profissional de evitar remoção excessiva de tecido sadio, acatando uma decisão minimamente invasiva, busca-se excelência estética. Sucesso estético este conseguido na escolha por esse tipo de restauração nos elementos dentários 11, 12, 13, 14, $15,21,22,23,24$, e 25 da paciente em questão.

Tratamentos restauradores conservadores diretos também podem ser usados para solucionar problemas funcionais, estéticos e anatômicos. Quesitos como idade do paciente, quantidade de estrutura dentária e custo serão importantes no momento da decisão de qual tratamento escolher ${ }^{11}$, outrossim, o baixo custo e mínimo desgaste de tecido dentário foram alguns dos quesitos que induziu à escolha por resinas compostas diretas.

Procedimentos estéticos que hoje são solucionados com tratamentos conservadores com uso de resinas compostas, antes só eram realizados com trabalhos protéticos mais invasivos ${ }^{12}$. Diante do avanço dos materiais dentários, a técnica restauradora de faceta com compósitos possibilitou que casos clínicos como o apresentado sejam possíveis de realização.

A escolha de resinas compostas e restaurações diretas leva em consideração o bom desempenho do material e ser uma técnica prática e reversível, como relatado por Wanderley et al. ${ }^{13}$. No caso descrito a praticidade da técnica foi um dos motivos para se optar pelas resinas compostas, corroborando com o autor.

Segundo Frese at al. ${ }^{14}$ ao se fazer um comparativo entre laminados cerâmicos e resinas compostas se observa que apesar dos laminados apresentarem maior durabilidade as resinas trazem consigo vantagens como: menor desgaste de estrutura dentária, ser uma técnica de fácil reversibilidade e apresentar menor custo. No caso descrito realizou-se apenas remoção das restaurações antigas, mínimos desgastes dentários e em alguns elementos não necessitou desgastar tecido dentário sadio, concordando com estudos encontrados na literatura a respeito da minimização dos desgastes.

Para Souza et al. ${ }^{15}$ apesar das vantagens dos compósitos resinosos o tratamento restaurador direto exige boa habilidade técnica do cirurgião-dentista. Dessa forma para se obter êxito no resultado final das restaurações diretas é necessário seguir passos importantes como, confecção de procedimentos préoperatórios, seleção do material, seleção de cor, isolamento eficaz, preparo dentário adequado, inserção correta dos incrementos resinosos para que se obtenha um resultado mais próximo do natural possível $^{16}$, no caso clínico supracitado, corroborou-se com os autores sobre todas essas etapas para conseguir um excelente resultado final.

Além de se observar detalhes dos dentes é importante analisar os tecidos periodontais adjacentes, no caso descrito a paciente apresentava coroas clínicas curtas. Como se sabe, o tratamento cirúrgico periodontal tem sido cada vez mais utilizado como um importante aliado na busca pela estética e harmonia do sorriso, principalmente em casos que envolvem dentes anteriores ${ }^{17}$.

Além disso, para o caso clínico descrito não teria sido possível um resultado estético tão satisfatório se as coroas clínicas curtas não tivessem sido corrigidas com o procedimento cirúrgico periodontal para aumento de coroa clínica. Para Cruz et al. ${ }^{18}$ o desempenho profissional mais consciente sobre a anatomia e habilidade manual, recuperando forma e função, fisiologia mastigatória, harmonia e estética entre os arcos é muito importante para o sucesso clínico. Sousa et al. ${ }^{19}$ destacam o comportamento clínico satisfatório verificado nas restaurações com resina composta.

\section{CONCLUSÃO}

A técnica restauradora com resina composta é uma opção de baixo custo e boa resolutividade que 
se mostrou uma excelente opção de tratamento para o caso relatado, pois respondeu bem aos objetivos de devolver saúde e reestabelecer a harmonia do sorriso.

\section{REFERÊNCIAS}

1. Marcondes R, Pires HCS, Bocutti JH. Lâmina cerâmica unitária sobre substrato escurecido: protocolo clinicolaboratorial com estratifcação em duas camadas. Rev Dental Press Estét. 2012;9(4):28-44.

2. Sakamoto Junior AS, Higashi C, Gomes JC. Substituição de coroas totais metalocerâmicas em incisivos laterais conoides vitais: relato de caso clínico. Rev Dental Press Estét. 2015;12(3):71-83.

3. Pontons-Melo JC. Harmonização do sorriso por meio de procedimentos minimamente invasivos. FGM News. 2013;132-37.

4. Mondelli J. Estética e cosmética: em clínica integrada restauradora. São Paulo: Quintessence; 2003.

5. Baratieri LN. Soluções clínicas: fundamentos e técnicas. Florianópolis: Ponto; 2008.

6. Silva SB. Facetas diretas de resina composta versus facetas indiretas em porcelana [monografia] Florianópolis: ABO-SC; 2005.

7. Queiroga RB. Laminados cerâmicos minimamente invasivos: novas possibilidades. Rev Dental Press Estét. 2012;9(1):34-47.

8. Soares PV, Faria NFB, Cardoso IO, Moura GF, Pereira AG. Multidisciplinary approach for rehabilitation of smile aesthetics with minimally invasive ceramic veneers. J Clin Dent Res. 2017;14(1):68-88.

9. Joiner A, Luo W. Tooth colour and whiteness: A review. J Dent. 2017; 67S:S3-S10.

10.Nash RW. Resurfacing tooth structure with ceramic laminates. Dent Today. 2014; 33(8):68-71.

11.Dietschi D. Optimizing smile composition and esthetics with resin composites and other conservative esthetic procedures. Eur J Esthet Dent. 2008;3(1):14-29.

12.Almeida RR, Garib D.G, Almeida-Pedrin RR, Almeida MR, Pinzan A, Junqueira MHZ. Diastemas interincisivos centrais superiores: quando e como intervir?. R Dental Press Ortodon Ortop Facial. 2004;9(3):137-56.

13.Lima RBW, Leite JT, França RM, Brito MCT, Uchôa RC, Andrade AKM. Reabilitação estética anterior pela técnica do facetamento - relato de caso. Rev bras ciênc saúde 2013; 17(4):363-70.

14.Frese C, Schiller P, Staehle HJ, Wolff D. Recontouring teeth and closing diastemas with direct composite buildups: A 5-year follow-up. J Dent. 2013;41(11):979-85.

15. Souza SJB, Magalhães D, Silva GR, Soares CJ, Soares PFB, Santos-Filho PCF. Cirurgia plástica periodontal para correção de sorriso gengival associada a restaurações em resina composta: relato de caso clínico. Rev Odontol Bras Central. 2010;19(51):362-66.

16.Ferreira CLB. Fraturas dentárias no sector anterior abordagem estética através de restaurações diretas a resina composta [dissertação]. Porto: Faculdade de Ciências da Saúde Universidade Fernando Pessoa; 2013.

17.Okida RC, Rahal V, Okida DSS. A associação entre dentística e periodontia no tratamento estético com lentes de contato: relato de caso. Rev Odontol Araçatuba. 2015;36(1):59-64.

18.Cruz JHA, Silva RLB, Andrade-Júnior FP, Guênes GMT, Almeida MSC, Medeiros LADM et al. A importância da anatomia e escultura dental para prática de procedimentos clínicos odontológicos. RSC online, 2018;7(1):76-85.

19. Sousa LX, Cruz JHA, Melo WOS, Freire SCP, Ribeiro ED, Freire JCP. Abfração dentária: um enfoque sobre a etiologia e o tratamento restaurador. Arch Health Invest. 2018; 7(2):51-53.

\section{CONFLITO DE INTERESSES}

Os autores declaram não haver conflitos de interesse.

\section{AUTOR PARA CORRESPONDENCIA}

\section{José Henrique de Araújo Cruz}

henrique_araujo1992@ hotmail.com

Submetido em 09/09/2018

Aceito em 12/03/2019 